\title{
App and web-based clinical communication solution and pager replacement
}

\author{
Authors: Anuja Bambaravanage* and Sudhir Singh
}

\section{Introduction}

Oxford University Hospital NHS Foundation Trust (OUHFT) is a large tertiary care NHS trust in the UK. It has an ageing pager system which has been in use for over 40 years. Currently, medical staff increasingly use unofficial encrypted messaging platforms not meeting the information governance requirements for effective pager, landline-free communication.

As medicine advances, health needs change and society develops, the NHS has to continually move forward so that in 10 years' time we have a service fit for the future. Virtually every aspect of modern life has been, and will continue to be, radically reshaped by innovation and technology - and healthcare is no exception. ${ }^{1}$

To ensure NHS systems and NHS data are secure through implementation of security, monitoring systems and staff education. Encourage a world leading health IT industry in England with a supportive environment for software developers and innovators.

Clinical communication solution is an ongoing quality improvement project undertaken as part of the Royal College of Physicians Chief Registrar Programme, in line with OUHFT's strategic themes.

\section{Materials and methods}

The first phase pilot was launched in February 2019 at Horton General Hospital, one of the trust's four sites. See Fig 1.

\section{Results and discussion}

Advantages:

> Savings - $€ 137,000$ per year by not renewing current pager system contract and more by not upgrading the ageing infrastructure (transmitters).

> Instant contact - no more waiting around for 'bleeps' to be answered, calling switchboard, phone ping-pong.

$>$ Know when a message has been read and received.

> Share photos/images/electrocardiogram and patient information with confidence - rather than risk breaching patient confidentiality by using unsuitable apps.

Authors: Oxford University Hospitals NHS Foundation Trust ${ }^{*} \mathrm{RCP}$ chief registrar

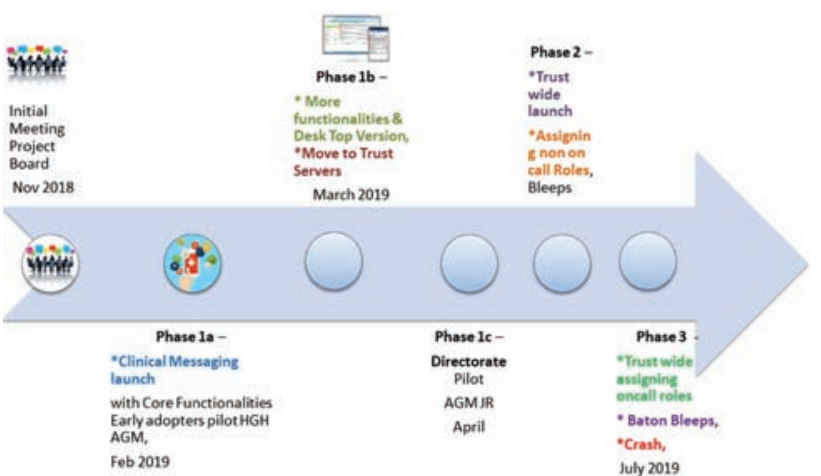

Fig 1. Phases of the clinical communication project

> Bleeps lack crucial detail, giving clinicians no prioritising of tasks that can be dealt with later.

$>$ Know who's on call without needing to reference black books and contact lists on wards, going to switchboard or bothering off-duty colleagues.

$>$ Easy, direct, contact and dialogue.

$>$ Use on the move.

$>$ Auditing, clinical resource management - diverting workforce to areas of need based on priority/demand and capacity.

$>$ Likelihood of the person who bleeped being busy at the time of a return call is high.

> The bleep system is also one-directional - forcing users to contact a specific individual. This individual may already be engaged on other time-consuming clinical activity and out of circulation. Meanwhile other clinicians who are able to respond immediately remain oblivious to the need.

$>$ This inability to manage tasks in a timely and appropriate fashion is costly at every level. Sub-optimal patient care can lead to unnecessary increases in lengths of stay, placing greater pressure on resources and depriving others of hospital care.

\section{Conclusion}

App and web-based clinical communication solution and pager replacement needs to keep pace with advances in digital technology to save financial and workforce resources and to make NHS ready for the future (Fig 2). 


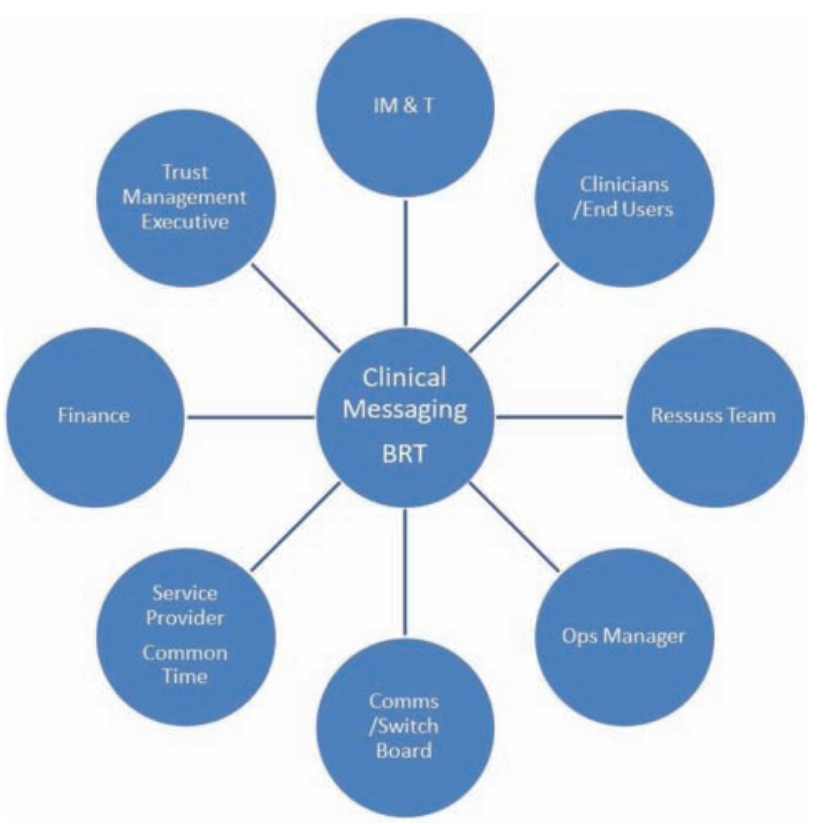

Fig 2. Areas that use clinical messaging

\section{Reference}

1 NHS. Long Term Plan. NHS England, 2019. 